\title{
Limited Efficacy of Adjuvant Therapy with Dexamethasone in Preventing Hearing Loss Due to Experimental Pneumococcal Meningitis in the Infant Rat
}

\author{
RONEY S. COIMBRA, GÉRARD LOQUET, AND STEPHEN L. LEIB \\ Institute for Infectious Diseases [R.S.C., S.L.L.], University of Bern, CH-3010 Bern, Switzerland; Department of Medicine [G.L.], \\ Unit of Physiology, University of Fribourg, CH-1700 Fribourg, Switzerland
}

\begin{abstract}
Sensorineural hearing loss (SNHL) is the most common sequel of bacterial meningitis (BM) and is observed in up to $30 \%$ of survivors when the disease is caused by Streptococcus pneumoniae. BM is the single most important origin of acquired SNHL in childhood. Anti-inflammatory dexamethasone holds promises as potential adjuvant therapy to prevent SNHL associated with BM. However, in infant rats, pneumococcal meningitis (PM) increased auditory brainstem response (ABR) thresholds [mean difference $=54$ decibels sound pressure level (dB SPL)], measured $3 \mathrm{wk}$ after infection, irrespective to treatment with ceftriaxone plus dexamethasone or ceftriaxone plus saline $(p<0.005$ compared with mock-infected controls). Moreover, dexamethasone did not attenuate short- and long-term histomorphologic correlates of SNHL. At $24 \mathrm{~h}$ after infection, blood-labyrinth barrier (BLB) permeability was significantly increased in infected animals of both treatment groups compared with controls. Three weeks after the infection, the averaged number of type I neurons per square millimeter of the Rosenthal's canal dropped from $0.3019 \pm 0.0252$ in controls to $0.2227 \pm 0.0635$ in infected animals receiving saline $(p<0.0005)$. Dexamethasone was not more effective than saline in preventing neuron loss $(0.2462 \pm 0.0399 ; p>0.05)$. These results suggest that more efficient adjuvant therapies are needed to prevent SNHL associated with pediatric PM. (Pediatr Res 62: 291-294, 2007)
\end{abstract}

$\mathrm{B}^{\mathrm{N}}$ $\mathrm{M}$, particularly in children, is associated with devastating mortality rates of up to $30 \%$. Despite appropriate therapy, $20 \%-50 \%$ of pediatric patients who survive the infection have permanent neurologic sequels, which include deafness, mental retardation, and learning impairment, sensorimotor deficits, seizure disorders, and cerebral palsy. Streptococcus pneumoniae is the most common cause of BM in infants and young children worldwide (1). Pneumococcal meningitis (PM) is consistently associated with a particularly high incidence of neurologic sequelae, with up to half of the survivors presenting some form of neurologic deficits $(2,3)$.

SNHL, usually profound and unilateral, is the most common neurologic sequel of BM and is observed in as many as $30 \%$ of survivors when the etiologic agent is S. pneumoniae $(4,5)$. BM is the single most important cause of

Received February 7, 2007; accepted April 16, 2007

Correspondence: Stephen L. Leib, M.D., Institute for Infectious Diseases, University of Bern, Friedbuhlstrasse 51, P.O. Box 61, CH-3010 Bern, Switzerland; e-mail: stephen.leib@ifik.unibe.ch

This work was supported by grants from the Swiss National Science Foundation (632-66057.01) and from the NIH (2P50NS035902-06). acquired SNHL in childhood despite appropriate antimicrobial therapy (6).

SNHL results from involvement of the inner ear due to inflammation as the cerebrospinal fluid (CSF) containing pneumococci and leukocytes extends from the subarachnoid space via the cochlear aqueduct to the perilymph (7-9). In an adult rat model of PM, the severity of permanent hearing impairment assessed $2 \mathrm{wk}$ after the infection correlated significantly with the loss of type I spiral ganglion neurons (10). We have recently confirmed and expanded the correlation between the loss of type I neurons in the spiral ganglion and the degree of hearing impairment in infant rats (11). Indeed, we have shown that adjuvant therapy with doxcycline combined with ceftriaxone significantly attenuated neuron loss in the spiral ganglion. Attenuation of neuron loss was accompanied by a moderate, yet statistically significant decrease in long-term ABR thresholds when compared with infected animals receiving only ceftriaxone and saline (11).

The contribution of excessive inflammation to the pathogenesis of brain and inner ear damage in BM has motivated a number of studies aiming to access the neuroprotective potential of adjuvant therapy with the anti-inflammatory drug dexamethasone. Some of these studies have proven the ability of dexamethasone to decrease the incidence and severity of SNHL in children with Haemophilus influenzae meningitis when given in combination with antibiotic therapy (12-14). However, a beneficial effect of adjuvant dexamethasone to infants with PM has not yet been demonstrated (15). Results from different surveys are controversial and not conclusive, in part because of the small number of patients with PM enrolled $(12,16-21)$.

Experimental studies evaluating the neuroprotective potential of adjuvant dexamethasone in PM have generated conflicting data. Whereas dexamethasone was shown to decrease neurologic sequels and caspase activity in an adult rat model of PM (22), it increased both acute hippocampal injury and long-term learning deficits in adult rabbits (23) and in infant rats (24). Experimental data on the potential of adjuvant

\footnotetext{
Abbreviations: ABRs, auditory brainstem responses; BM, bacterial meningitis; CFUs, colony-forming units; CSF, cerebrospinal fluid; dB SPL, decibels sound pressure level; OD, optical density; PM, pneumococcal meningitis; SNHL, sensorineural hearing loss
} 
dexamethasone to prevent inner ear damage associated with PM is scarce and limited to adult animal models $(25,26)$. To our knowledge, the present report is the first experimental study assessing the potential of dexamethasone to prevent hearing loss in an infant model of PM.

\section{METHODS}

Model of meningitis with live pneumococci. Nursing Wistar rats were injected intracisternally on postnatal d 11 (P11) with $10 \mu \mathrm{L}$ of saline containing $2.5 \times 10^{6} \pm 1.5 \times 10^{6}$ colony-forming units $(\mathrm{CFUs}) / \mathrm{mL} S$. pneumoniae as described previously (27). At $18 \mathrm{~h}$ after infection, all animals were assessed clinically as described elsewhere $(24,27)$, and CSF was obtained from the cisterna magna to document infection and to determine the number of bacteria by plating serial dilutions of CSF on blood agar plates. If incubation of $5 \mu \mathrm{L}$ of undiluted CSF did not result in growth of bacteria, the CSF was considered to be sterile and animals were excluded from further analysis. The detection limit for bacteria in the CSF was $200 \mathrm{CFUs} / \mathrm{mL}$.

All animal experiments were approved by the Animal Care and Experimentation Committee of the Canton of Bern, Switzerland, and were performed using National Institutes of Health guidelines for animal experiments.

Assessment of BLB integrity in acute PM. To assess early damage to the cochlea during acute PM, animals were infected $(n=7)$ and randomized for treatment with dexamethasone (Fortecortin, Merck, Dietikon, Switzerland) [one dose of $0.7 \mathrm{mg} / \mathrm{kg}$ i.p. at $18 \mathrm{~h}$ after infection, i.e. together with the first dose of antibiotics $(n=4)$ ] or with the same volume of saline $(n=3)$. The dose of $0.7 \mathrm{mg} / \mathrm{kg}$ used in the study corresponds to the dose used in earlier studies on adjuvant dexamethasone in experimental pneumococcal meningitis in infant rats (24). The higher dose of dexamethasone in comparison with the dose used in infants (usual dose in children: $0.15 \mathrm{mg} / \mathrm{kg}$ body weight) was chosen to compensate for the higher clearance rates of infant rats (28). Mock-infected control animals $(n=3)$ were also given dexamethasone. All animals $(n=10)$ were treated with one single dose of $100 \mathrm{mg} / \mathrm{kg}$ ceftriaxone s.c. (Roche Pharma, Reinach, Switzerland) at $18 \mathrm{~h}$ after infection. Twentythree hours after infection, animals were injected with $1 \mathrm{~mL} 1 \%$ Evans blue i.p. and killed $1 \mathrm{~h}$ later. Evans blue was quantified in cochlear histologic sections prepared as describe above by fluorescence microscopy using a 454-nm excitation filter and a 590-nm barrier filter (29). Three pictures showing different regions of the stria vascularis (i.e. lower, mid, and upper cochlear turns) were captured with a CDD imaging system (Hamamatsu Photonics, Solothurn, Switzerland), and the optical density (OD) per square millimeter of the stria vascularis was quantified using the NIH Image program (version 1.61; U.S. National Institutes of Health, Bethesda, MD). The ODs per square millimeter were adjusted by subtracting the OD of the area corresponding to the stria vascularis from the OD of the background. The adjusted ODs were averaged per cochlea $(10,30)$.

Assessment of long-term damage to the cochlea. To assess long-term cochlear damage, animals were infected $(n=29)$ and randomized for treatment with dexamethasone ( 12 doses of $0.7 \mathrm{mg} / \mathrm{kg}$ i.p. every $8 \mathrm{~h} ; n=17$ ) starting at $18 \mathrm{~h}$ after infection, together with the first dose of antibiotics, or with the same volume of saline $(n=12)$. Mock-infected control animals $(n=$ 13) were also treated with dexamethasone. All animals $(n=42)$ were treated with eight doses of $100 \mathrm{mg} / \mathrm{kg}$ ceftriaxone s.c. (Roche Pharma, Reinach, Switzerland) every $12 \mathrm{~h}$. This treatment schedule was maintained for $4 \mathrm{~d}$, starting $18 \mathrm{~h}$ after infection. At $3 \mathrm{wk}$ after infection, the ABR were measured in both ears of a subset of randomly chosen animals $(n=14, n=9$, and $n=$ 6 for the dexamethasone, saline, and mock-infected groups, respectively), and then the animals were killed. The density of type I neurons in the spiral ganglion was assessed by histomorphologic analysis of cochlear sections in one randomly chosen cochlea per animal.

We ran two separate pilot studies to set up the methods of cochlear histomorphology and ABR measurements. Only in the final study, animals were tested with both methods. Because the histomorphologic analysis and ABR measurements were conducted with the same protocol in both the pilot and final studies, we included both results in this report. Only animals from the final study are represented in the correlation analysis (see "Statistics" below).

Histomorphologic analysis of cochlear sections. To determine the density of type I neurons in the spiral ganglion, the region of the temporal bone containing the cochlea was dissected from the skull, postfixed for $4 \mathrm{~h}$ in paraformaldehyde, and decalcified for $10 \mathrm{~d}$ in phosphate-buffered saline containing $120 \mathrm{mM}$ ethylenediaminetetraacetic acid and $0.1 \%$ sodium azide. One cochlea of each animal was embedded in paraffin, and the six most mid-modiolar sections $(10 \mu \mathrm{m}$ thick) were mounted on glass slides and stained with toluidine blue. The specimens were analyzed with a light microscope, and the images were digitized. The outline of the Rosenthal's canal was traced using the NIH Image program. The type I neurons in 10 profiles of the Rosenthal's canal, from the base to the apex of the cochlea, were counted $(11,31)$. The results were expressed as the density of type I neurons per square millimeter. All histopathologic evaluations were done by an investigator who was unaware of the clinical, microbiologic, and treatment status of the animal.

ABR measurements. ABRs were recorded for inactin-anesthetized rats 3 wk after infection (11). Measurements were performed in an audiometric room (model AC-1 chamber; Industrial Acoustics Company, Niederkrüchten, Germany) for both ears in response to click stimulation (presentation rate, 20 clicks/s). Subdermal needle electrodes were placed in the mastoid of the tested ear (active), at the vertex (reference), and in the cervical neck muscles (ground). The sound stimulus consisted of $100 \mu$ s square wave impulses synthesized digitally using SigGen32 software (System II; Tucker- Davis Technologies, Alachua, FL), fed into a programmable attenuator, and after D/A conversion transduced by a speaker $(2405 \mathrm{H}$ ultra-high-frequency driver; JBL Professional, Northridge, CA) located $4 \mathrm{~cm}$ from the pinna. The system was calibrated with a sound level meter (2231 modular precision sound level meter; Brüel \& Kjær, Nærum, Denmark) by measuring with a 0.5 -in. microphone (type 4189; Brüel \& Kjær, Nærum, Denmark) the maximum peak level (expressed in $\mathrm{dB}$ SPL; reference pressure, $20 \mu \mathrm{Pa}$ ) emitted by the speaker when it was driven by a square wave impulse. ABR were amplified $\left(10^{3}\right)$, band-pass filtered between $100 \mathrm{~Hz}$ and $3 \mathrm{kHz}$ (model 1201 low-noise preamplifier; Ithaco, Ithaca, NY), and averaged $(n=500)$ during a $12-\mathrm{ms}$ window with the data acquisition software BioSig32 (System II; Tucker-Davis Technologies, Alachua, FL). The intensity of the click stimulus was reduced from 110 to $0 \mathrm{~dB}$ SPL in 10-dB steps. The hearing threshold was defined as the lowest intensity, which induced the appearance of a visually detectable first peak. The ABR responses of both ears of each animal were averaged.

Statistics. Normally distributed variables were presented as mean \pm SD unless stated otherwise and were compared using one-way analysis of variance followed by an unpaired $t$ test. Variables that were not normally distributed (hearing thresholds) were compared using the Kruskal-Wallis test. When the latter yielded a statistically significant value $(p<0.05)$, pairwise comparison was done using the two-tailed nonparametric Mann-Whitney $U$ test. Correlation between ABR thresholds and density of type I neurons in the spiral ganglion was tested by nonparametric correlation (Spearman).

\section{RESULTS}

Evans blue extravasation into the stria vascularis. At $24 \mathrm{~h}$ after infection, Evans blue extravasation into the stria vascularis, which is an indication of BLB disruption, was significantly increased $(p<0.05)$ in infected animals $(7.84 \pm 0.95)$ compared with mock-infected controls $(2.81 \pm 1.77)$. Adjuvant treatment with one single dose of dexamethasone given at $18 \mathrm{~h}$ after infection failed to attenuate BLB disruption (9.75 \pm 2.00; $p<0.01$ compared with vehicle-treated animals).

Loss of neurons in the spiral ganglion. Pneumococcal meningitis caused significant loss of type I neurons in the spiral ganglion, as determined $3 \mathrm{wk}$ after the infection. The averaged density of type I neurons per square millimeter of the Rosenthal's canal was $0.3019 \pm 0.0252$ for mock-infected animals versus $0.2227 \pm 0.0635$ for animals that were infected and treated with ceftriaxone plus saline $(p<0.0005)$. Adjuvant dexamethasone did not attenuate the loss of type I neurons in infected animals $(0.2462 \pm 0.0399)$ compared with treatment with saline and ceftriaxone $(p>0.05)$ (Fig. 1A). Infected animals receiving dexamethasone had significantly fewer type I neurons in their spiral ganglia compared with mock-infected animals $(p=0.0001)$.

ABR measurements. PM caused a significant increase in $\mathrm{ABR}$ thresholds (mean difference $=54 \mathrm{~dB}$ SPL) determined 3 wk after infection $[100 \pm 11.73$ for ceftriaxone and salinetreated infected animals versus $45.83 \pm 14.63$ for ceftriaxone and dexamethasone-treated mock-infected animals $(p<$ 0.0005)]. Adjuvant dexamethasone did not prevent the in- 

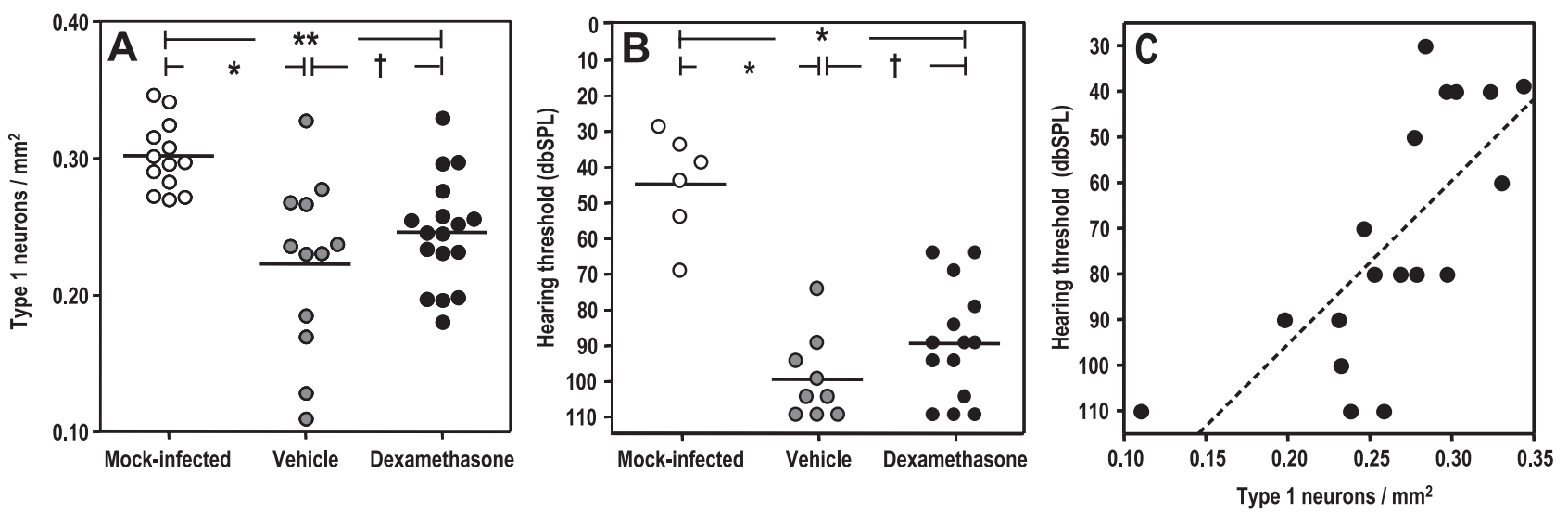

Figure 1. Anatomical and functional changes associated with hearing impairment at $3 \mathrm{wk}$ after experimental PM or mock infection. (A) PM caused a significant loss of type I neurons in the spiral ganglion of infected animals treated with saline $(* p<0.0005 ; n=12)$ and dexamethasone $(* * p<0.0001 ; n=17)$ when compared with mock infection ( $n=13, n$ refers to the number of animals). One randomly chosen cochlea was analyzed per each animal. Adjuvant therapy with dexamethasone had no significant effect on density of type I neurons in the Rosenthal's canal. $\dagger$ Not significant. $(B)$ PM led to a significant increase in ABR thresholds $3 \mathrm{wk}$ after infection irrespective of adjuvant treatment regimen $\left({ }^{*} p<0.0005\right.$ for both treatment groups) when compared with ABR thresholds in mock-infected animals $(n=6)$. Adjuvant dexamethasone did not prevent the increase in ABR thresholds in infected animals compared with treatment with ceftriaxone and saline ( $\dagger p=\mathrm{ns}(>0.05), n=14$ and 9, respectively). The ABR responses of both ears of each animal were averaged. In our experimental conditions, a 110-dB hearing loss value represents deafness. (C) A significant negative correlation (Spearman $r=-0.78 ; p=0.0001 ; n=18$, one ear per animal) was found between hearing thresholds and the number of type I neurons in the Rosenthal's canal. Linear regression curve defined by $y=167.2-358.6 x$. Points in Figure $1 C$ represent ears that have been analyzed by both ABR and cochlear histomorphology $(n=18$ animals, one ear per animal).

crease in ABR thresholds in infected animals $(90 \pm 15.81)$ compared with treatment with ceftriaxone and saline $(100 \pm$ $11.73 ; p>0.05$ ) (Fig. $1 B$ ).

The density of type I neurons in the spiral ganglion correlated negatively with ABR thresholds (Spearman $r=-0.78$; $p=0.0001$, Fig. 1C).

\section{DISCUSSION}

Anti-inflammatory therapy with adjuvant dexamethasone has been regarded with optimism as a feasible strategy to prevent ear damage associated with BM. Several successful clinical trials have shown that adjuvant dexamethasone improves hearing outcome in children with $H$. influenzae meningitis (12-14). However, data from clinical trials available at present do not allow any conclusion on the potential benefit of adjuvant dexamethasone to prevent SNHL in children with PM $(12,14,16-19,21)$. Experimental data on animal models of PM are scarce and limited to adults $(25,26)$. None of them attempted to investigate the morphologic correlates of hearing loss. In an adult rabbit model of PM, the administration of dexamethasone $10 \mathrm{~min}$ before ampicillin (therapeutic regimen starting at $18 \mathrm{~h}$ after infection) attenuated SNHL compared with placebo, as measured by ABR $8 \mathrm{~d}$ after infection (26). More recently, it was reported that adjuvant dexamethasone preserved cochlear function in an adult gerbil model of PM (25). In this report, ABR measurements were performed 3 months after infection. Altogether, these findings suggest a beneficial role of dexamethasone in attenuating long-term and irreversible hearing loss associated with PM in adult animals, provided that therapy is initiated before or at the time of initiation of the antibiotic therapy (15).

In agreement with previous studies using adults rats $(10,30)$, we found herein that, in infant rats, PM induces a pronounced disruption of the BLB, as evidenced by Evans blue extravasation into the stria vascularis. Sensory transduction in the mammalian cochlea requires the homeostasis of specialized fluid compartments with distinct ionic compositions. The stable composition of endolymph is achieved by the concerted action of diverse ion channels and transporters, most located at the stria vascularis, and by an integral BLB $(32,33)$. Thus, it seems reasonable to think that disruption of the BLB during the acute phase of PM may contribute to the cochlear dysfunction. However, the present investigation does not allow further insights into the toxic mechanism. Nevertheless, the present study shows that adjuvant dexamethasone failed to preserve the integrity of the BLB during acute PM.

We assessed short- and long-term cochlear damage associated with PM at the very onset of hearing in infant rats, i.e. infection on P11. In the rodent cochlea, stereocilia sprout from the apical surface of sensory hair cells at embryonic d 15 and the hair bundles reach their final adult shape by P6. The organ of Corti reaches its adult size by P10, becomes functional at around $\mathrm{P} 12-\mathrm{P} 14$, and is considered morphologically and functionally mature at P21 $(34,35)$. The onset of hearing (P12P14) is associated with increased ionic and metabolic activity and with changes in cell proliferation and growth. We demonstrated in our study that dexamethasone is not effective in preventing SNHL in infant rats experimentally infected with S. pneumoniae at P11. Our results contrast with those of a previous report of SNHL attenuation by adjuvant dexamethasone in adult rats with PM (26). The differential response to dexamethasone in the adult and the infant rat model needs to be further investigated, but it may be due to the metabolic and growth changes taking place in the immature cochlea at the time of hearing onset $(34,35)$. We have also shown here that adjuvant therapy with dexamethasone did not prevent damage to cochlear structures, namely, the stria vascularis and the spiral ganglion neurons in PM. A strong correlation was 
observed between the density of type I neurons in the spiral ganglion and the shifts in the ABR thresholds, which confirms the hypothesis that loss of this subpopulation of neurons is an important morphologic correlate of long-term hearing loss associated with PM $(10,11)$. The present results demonstrate the failure of dexamethasone to prevent SNHL in the infant rat, contrary to previously successful trials in adult animal models $(25,26)$. A direct comparison with the data generated in the adult model is not possible because the effects of dexamethasone on morphologic correlates of SNHL have not been reported $(25,26)$. It is important to note that the results of the above-mentioned studies with adult animals disagree with the conclusions of the European Dexamethasone Adulthood Bacterial Meningitis Study Investigators that in adult human patients with PM, adjuvant dexamethasone did not have a significant beneficial effect on neurologic sequels, including hearing loss $(36,37)$.

In conclusion, adjuvant therapy with dexamethasone failed to prevent SNHL and short- and long-term damage to cochlear structures in an infant rat model of PM. The present results suggest that more efficient adjuvant therapies are needed to prevent hearing loss associated with PM in the developing ear.

Acknowledgments. The authors thank Jürg Kummer, Philipp Joss, and Franziska Simon for excellent technical support.

\section{REFERENCES}

1. Yogev R, Guzman-Cottrill J 2005 Bacterial meningitis in children: critical review of current concepts. Drugs 65:1097-1112

2. Hoogman M, van de Beek D, Weisfelt M, de Gans J, Schmand B 2007 Cognitive outcome in adults after bacterial meningitis. J Neurol Neurosurg Psychiatry [Epub ahead of print]

3. Merkelbach S, Sittinger H, Schweizer I, Muller M 2000 Cognitive outcome after bacterial meningitis. Acta Neurol Scand 102:118-123

4. Dodge PR, Davis H, Feigin RD, Holmes SJ, Kaplan SL, Jubelirer DP, Stechenberg BW, Hirsh SK 1984 Prospective evaluation of hearing impairment as a sequela of acute bacterial meningitis. N Engl J Med 311:869-874

5. Oostenbrink R, Maas M, Moons KG, Moll HA 2002 Sequelae after bacterial meningitis in childhood. Scand J Infect Dis 34:379-382

6. Fortnum HM 1992 Hearing impairment after bacterial meningitis: a review. Arch Dis Child 67:1128-1133

7. Dichgans M, Jager L, Mayer T, Schorn K, Pfister HW 1999 Bacterial meningitis in adults: demonstration of inner ear involvement using high-resolution MRI. Neurology 52:1003-1009

8. Bhatt SM, Lauretano A, Cabellos C, Halpin C, Levine RA, Xu WZ, Nadol JB Jr, Tuomanen E 1993 Progression of hearing loss in experimental pneumococcal meningitis: correlation with cerebrospinal fluid cytochemistry. J Infect Dis 167:675683

9. Bhatt S, Halpin C, Hsu W, Thedinger BA, Levine RA, Tuomanen E, Nadol JB Jr 1991 Hearing loss and pneumococcal meningitis: an animal model. Laryngoscope 101:1285-1292

10. Klein M, Koedel U, Pfister HW, Kastenbauer S 2003 Morphological correlates of acute and permanent hearing loss during experimental pneumococcal meningitis. Brain Pathol 13:123-132

11. Meli DN, Coimbra RS, Erhart DG, Loquet G, Bellac CL, Täuber MG, Neumann U, Leib SL 2006 Doxycycline reduces mortality and injury to the brain and cochlea in experimental pneumococcal meningitis. Infect Immun 74:3890-3896
12. Girgis NI, Farid Z, Mikhail IA, Farrag I, Sultan Y, Kilpatrick ME 1989 Dexamethasone treatment for bacterial meningitis in children and adults. Pediatr Infect Dis J 8:848-851

13. Lebel MH, Freij BJ, Syrogiannopoulos GA, Chrane DF, Hoyt MJ, Stewart SM, Kennard BD, Olsen KD, McCracken GH Jr 1988 Dexamethasone therapy for bacterial meningitis. Results of two double-blind, placebo-controlled trials. N Engl J Med 319:964-971

14. Odio CM, Faingezicht I, Paris M, Nassar M, Baltodano A, Rogers J, Saez-Llorens X, Olsen KD, McCracken GH Jr 1991 The beneficial effects of early dexamethasone administration in infants and children with bacterial meningitis. N Engl J Med 324:1525-1531

15. Grandgirard D, Leib SL 2006 Strategies to prevent neuronal damage in paediatric bacterial meningitis. Curr Opin Pediatr 18:112-118

16. Arditi M, Mason EO Jr, Bradley JS, Tan TQ, Barson WJ, Schutze GE, Wald ER, Givner LB, Kim KS, Yogev R, Kaplan SL 1998 Three-year multicenter surveillance of pneumococcal meningitis in children: clinical characteristics, and outcome related to penicillin susceptibility and dexamethasone use. Pediatrics 102:1087-1097

17. Kanra GY, Ozen H, Secmeer G, Ceyhan M, Ecevit Z, Belgin E 1995 Beneficial effects of dexamethasone in children with pneumococcal meningitis. Pediatr Infect Dis J 14:490-494

18. Kennedy WA, Hoyt MJ, McCracken GH Jr 1991 The role of corticosteroid therapy in children with pneumococcal meningitis. Am J Dis Child 145:1374-1378

19. McIntyre PB, Berkey CS, King SM, Schaad UB, Kilpi T, Kanra GY, Perez CM 1997 Dexamethasone as adjunctive therapy in bacterial meningitis. A meta-analysis of randomized clinical trials since 1988. JAMA 278:925-931

20. Prober CG 1995 The role of steroids in the management of children with bacterial meningitis. Pediatrics 95:29-31

21. Schaad UB, Kaplan SL, McCracken GH Jr 1995 Steroid therapy for bacterial meningitis. Clin Infect Dis 20:685-690

22. Irazuzta J, Pretzlaff RK, DeCourten-Myers G, Zemlan F, Zingarelli B 2005 Dexamethasone decreases neurological sequelae and caspase activity. Intensive Care Med 31:146-150

23. Zysk G, Bruck W, Gerber J, Bruck Y, Prange HW, Nau R 1996 Anti-inflammatory treatment influences neuronal apoptotic cell death in the dentate gyrus in experimental pneumococcal meningitis. J Neuropathol Exp Neurol 55:722-728

24. Leib SL, Heimgartner C, Bifrare YD, Loeffler JM, Täuber MG 2003 Dexamethasone aggravates hippocampal apoptosis and learning deficiency in pneumococcal meningitis in infant rats. Pediatr Res 54:353-357

25. Addison J, Kim HH, Richter CP 2006 Cochlear preservation after meningitis: an animal model confirmation of adjunctive steroid therapy. Laryngoscope 116:279282

26. Rappaport JM, Bhatt SM, Burkard RF, Merchant SN, Nadol JB Jr 1999 Prevention of hearing loss in experimental pneumococcal meningitis by administration of dexamethasone and ketorolac. J Infect Dis 179:264-268

27. Leib SL, Clements JM, Lindberg RL, Heimgartner C, Loeffler JM, Pfister LA, Täuber MG, Leppert D 2001 Inhibition of matrix metalloproteinases and tumour necrosis factor alpha converting enzyme as adjuvant therapy in pneumococcal meningitis. Brain 124:1734-1742

28. Spreer A, Gerber J, Hanssen M, Schindler S, Hermann C, Lange P, Eiffert H, Nau R 2006 Dexamethasone increases hippocampal neuronal apoptosis in a rabbit model of Escherichia coli meningitis. Pediatr Res 60:210-215

29. Chang-Ling T, Neill AL, Hunt NH 1992 Early microvascular changes in murine cerebral malaria detected in retinal wholemounts. Am J Pathol 140:1121-1130

30. Kastenbauer S, Klein M, Koedel U, Pfister HW 2001 Reactive nitrogen species contribute to blood-labyrinth barrier disruption in suppurative labyrinthitis complicating experimental pneumococcal meningitis in the rat. Brain Res 904:208-217

31. Shinohara T, Bredberg G, Ulfendahl M, Pyykko I, Olivius NP, Kaksonen R, Lindstrom B, Altschuler R, Miller JM 2002 Neurotrophic factor intervention restores auditory function in deafened animals. Proc Natl Acad Sci U S A 99:1657-1660

32. Osborne MP, Comis SD, Tarlow MJ, Stephen J 1995 The cochlear lesion in experimental bacterial meningitis of the rabbit. Int J Exp Pathol 76:317-330

33. Juhn SK 1988 Barrier systems in the inner ear. Acta Otolaryngol Suppl 458:79-83

34. Geal-Dor M, Freeman S, Li G, Sohmer H 1993 Development of hearing in neonatal rats: air and bone conducted ABR thresholds. Hear Res 69:236-242

35. Nishida Y, Rivolta MN, Holley MC 1998 Timed markers for the differentiation of the cuticular plate and stereocilia in hair cells from the mouse inner ear. J Comp Neurol 395:18-28

36. Weisfelt M, Hoogman M, van de Beek D, de Gans J, Dreschler WA, Schmand BA 2006 Dexamethasone and long-term outcome in adults with bacterial meningitis. Ann Neurol 60:456-468

37. de Gans J, van de Beek D 2002 Dexamethasone in adults with bacterial meningitis N Engl J Med 347:1549-1556 